\title{
FORÇA MUSCULAR E ÍNDICE DE FADIGA DOS EXTENSORES \\ E FLEXORES DO JOELHO DE JOGADORES PROFISSIONAIS \\ DE FUTEBOL DE ACORDO COM O \\ POSICIONAMENTO EM CAMPO
}

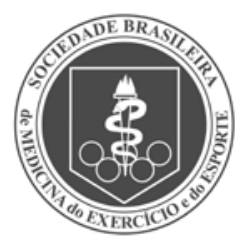

Artigo Original

\author{
MUSCULAR STRENGTH AND FATIGUE INDEX OF KNEE EXTENSORS AND FLEXORS OF \\ PROFESSIONAL SOCCER PLAYERS ACCORDING TO THEIR POSITIONING IN FIELD \\ FUERZA MUSCULAR E ÍNDICE DE FATIGA DE LOS EXTENSORES Y FLEXORES DE LAS RODILLAS DE \\ JUGADORES PROFESIONALES DE FÚTBOL, DE ACUERDO CON EL POSICIONAMENTO EN CAMPO
}

Viviane Otoni do Carmo

Carvalhais (Fisioterapeuta)

Thiago Ribeiro Teles dos Santos

(Fisioterapeuta) ${ }^{1}$

Vanessa Lara Araújo

(Fisioterapeuta) $^{1}$

Diego Xavier Leite

(Fisioterapeuta) ${ }^{1}$

João Marcos Domingues Dias

(Fisioterapeuta) $^{1}$

Sérgio Teixeira da Fonseca

(Fisioterapeuta)

1 Departamento de Fisioterapia; Escola de Educação Física, Fisioterapia e Terapia Ocupacional (EEFFTO), Universidade Federal de Minas Gerais (UFMG) - Belo Horizonte, MG, Brasil.

\section{Correspondência:}

Sérgio Teixeira da Fonseca

Escola de Educação Física,

Fisioterapia e Terapia Ocupacional -

Universidade Federal de Minas Gerais

Av. Presidente Antônio Carlos, 6.627

31270-901 - Belo Horizonte,

MG, Brasil.

sergioteixeirafonseca@gmail.com

\section{RESUMO}

Introdução: Assimetrias na capacidade de produção de força entre músculos dos membros inferiores e fadiga muscular podem favorecer a ocorrência de lesões em atletas de futebol. Considerando-se que existem diferenças individuais determinadas pelas diversas funções exercidas pelos jogadores, é possível que a presença de assimetrias de força e fadiga muscular esteja relacionada ao posicionamento em campo. Objetivos: 1) Investigar diferenças na assimetria de pico de torque (PT), na assimetria de trabalho (T) e no índice de fadiga (IF) dos extensores e flexores do joelho de atletas profissionais de futebol de acordo com a posição em campo; e 2) Determinar se o IF dos flexores é superior ao dos extensores. Métodos: Foram analisadas avaliações isocinéticas de 164 atletas profissionais de futebol (atacantes, zagueiros, laterais, meio-campistas e goleiros). O protocolo para avaliação da força concêntrica dos extensores e flexores do joelho consistiu em cinco repetições a $60 \%$ s e 30 repetições a $300 \%$ s. O teste de Kruskall-Wallis foi utilizado para verificar diferenças na assimetria de PT, assimetria de T e IF dos extensores e flexores do joelho entre jogadores de diferentes posicionamentos. $\mathrm{O}$ teste de Wilcoxon foi realizado para verificar se havia diferença entre o IF dos extensores e flexores. Resultados: Não houve diferença entre os jogadores dos cinco posicionamentos para as assimetrias de PT e T, bem como para o IF dos extensores e flexores ( $p>0,05)$. O IF flexor foi superior ao extensor em ambos os membros inferiores $(p<0,01)$. Conclusão: Variáveis isocinéticas comumente associadas a lesões não foram diferentes entre jogadores de diferentes posicionamentos. Os atletas apresentaram o IF flexor superior ao extensor, o que pode estar relacionado à maior frequência de estiramentos dos isquiossurais em comparação ao quadríceps.

Palavras-chave: atletas, dinamômetro de força muscular, membros inferiores.

\section{ABSTRACT}

Introduction: Strength asymmetry among the muscles of the lower limbs and muscle fatigue may predispose soccer players to injuries. Regarding the individual differences determined by diverse roles performed by players, it is possible that the presence of asymmetries of muscle strength and fatigue is related to playing position. Objectives: 1) To investigate differences in asymmetry of peak torque (PT), work asymmetry (W) and fatigue index (FI) of knee extensors and flexors of professional soccer players according to their positioning in field; 2) To determine if $\mathrm{Fl}$ of knee flexors is higher than those of knee extensors. Methods: Data from isokinetic assessment of 164 professional soccer players (forwards, fullbacks, wingers, midfielders and goalkeepers) were analyzed. The protocol of evaluation of concentric strength of knee extensors and flexors consisted of five repetitions at $60 \%$ and 30 repetitions at $300 \%$. Kruskall-Wallis test was carried out to verify differences in PT asymmetry, W asymmetry and $\mathrm{Fl}$ of knee extensors and flexors among players of different positions. The Wilcoxon test was performed to verify if there is difference between Fl of knee extensors and flexors. Results: There was no difference among playing positions for asymmetries of PT and $W$, as well as for Fl of knee extensors and flexors ( $p>0.05)$. The Fl of knee flexors was higher than Fl of knee extensors in both lower limbs $(p<0.01)$. Conclusion: Isokinetic variables, commonly associated with injuries, were not different among players of different positions. The athletes had flexor $\mathrm{Fl}$ higher than extensor $\mathrm{Fl}$, which can be related to greater frequency of strain injury of hamstrings in comparison to quadriceps.

Keywords: athletes, muscle strength dynamometer, lower extremity. 


\section{RESUMEN}

Introducción: Asimetrías en la capacidad de producción de fuerza entre músculos de los miembros inferiores y la fatiga muscular pueden favorecer la ocurrencia de lesiones en atletas de fútbol. Considerándose que existen diferencias individuales, determinadas por las diversas funciones desempeñadas por los jugadores, es posible que la presencia de asimetrías de fuerza y fatiga muscular esté relacionada con el posicionamiento en campo. Objetivos: 1) Investigar diferencias en la asimetría de pico de torsión (PT), en la asimetría de trabajo (T) y en el índice de fatiga (IF) de los extensores y flexores de las rodillas de atletas profesionales de fútbol, de acuerdo con la respectiva posición en campo; y 2) Determinar si el IF de los flexores es superior al de los extensores. Métodos: Se analizaron evaluaciones de 164 atletas profesionales de fútbol (delanteros, zagueros, laterales, mediocampistas y arqueros). El protocolo, para evaluación de la fuerza concéntrica de los extensores y flexores de las rodillas, consistió en cinco repeticiones a $60 \%$ y 30 repeticiones a $300 \%$ s. La prueba de Kruskall-Wallis fue utilizada para verificar diferencias en la asimetría de PT, asimetría de T e IF de los extensores y flexores de rodillas entre jugadores de diferentes posicionamientos. La prueba de Wilcoxon fue realizada para verificar si había diferencia entre el IF de los extensores y flexores. Resultados: No hubieron diferencias entre los jugadores de las cinco posiciones cuanto a las asimetrías de PT y T, así como para el IF de los extensores y flexores ( $p>0,05)$. El IF del flexor fue superior al del extensor en ambos miembros inferiores $(p<0,01)$. Conclusión: Las variables isocinéticas, comúnmente vinculadas a lesiones, no fueron diferentes entre jugadores en diversas posiciones. Los atletas presentaron el IF de flexor superior al del extensor, lo que puede estar relacionado con más frecuencia de estiramientos de los isquiosurales en comparación con el cuádriceps.

Palabras clave: atletas, dinamómetro de fuerza muscular, miembros inferiores.

\section{INTRODUÇÃO}

O futebol envolve movimentos que impõem carga assimétrica sobre membros inferiores (MMII), tais como chute e manobras de mudança de direção' ${ }^{1}$. Os atletas profissionais de futebol geralmente apresentam um membro inferior de preferência para realização desses movimentos ${ }^{1,2}$. A prática desse esporte, portanto, pode favorecer a ocorrência de assimetrias de força entre MMII, aumentando a suscetibilidade do atleta a lesões ${ }^{3-8}$. Intervenções que reduzam a assimetria na capacidade de produção de força podem resultar em menor incidência de lesões ${ }^{6}$. Assim, a investigação da assimetria de força entre MMII deve ser considerada nas avaliações pré-temporada, auxiliando no desenvolvimento de programas preventivos.

Apesar de alguns estudos demonstrarem a ocorrência de assimetrias em atletas de futebo| $2,9,11$, outros trabalhos não suportam esses achados $^{1,10,12}$. Considerando as diferenças no gesto esportivo determinadas pelas diversas funções exercidas pelos jogadores, é possível que a presença de assimetria nesses atletas esteja relacionada ao posicionamento em campo. Poucos estudos realizaram essa investigação, sendo que apenas três posicionamentos são geralmente considerados (defensores, meio-campistas e atacantes) ${ }^{13,14}$. No entanto, atletas que atuam pelas laterais do campo podem necessitar de maior quantidade de movimentos assimétricos em comparação a outros posicionamentos. Portanto, é provável que esse grupo de atletas tenha maior déficit de força entre os MMII. Assim, não considerar a posição em campo dos atletas na avaliação das assimetrias, ou não incluir separadamente a categoria de jogadores que atuam pelas laterais do campo, pode justificar os resultados ainda controversos.

A variável pico de torque é comumente utilizada para investigar a assimetria de força entre MMII2,9,10. Apesar disso, o trabalho muscular é outro parâmetro que pode ser útil na avaliação do desempenho muscular ${ }^{15}$. Essa variável reflete a capacidade da musculatura em gerar torque em toda amplitude de movimento e está relacionada à energia despendida pelo músculo durante o teste ${ }^{15,16}$. Assim, incluir a investigação do trabalho muscular em avaliações isocinéticas pode fornecer informações adicionais sobre a presença de assimetria de força entre os MMII.
A avaliação isocinética, dentre outros testes, é capaz de fornecer informação sobre a fadiga muscular (índice de fadiga), quando o protocolo selecionado envolve um grande número de repetições ${ }^{17}$. A fadiga dos músculos que cruzam a articulação do joelho parece contribuir para a ocorrência de ruptura do ligamento cruzado anterior ${ }^{18}$. Além disso, lesões por estiramento dos isquiossurais apresentam maior incidência no segundo tempo de uma partida de futebol, sugerindo que a fadiga muscular pode ser um dos fatores de risco para a ocorrência dessas lesões ${ }^{19}$. A frequência de estiramento dos flexores do joelho é maior quando comparada à dos extensores ${ }^{20,21}$, o que poderia ser justificado por uma maior fatigabilidade dos isquiossurais. Além disso, considerando que as características do treinamento e as atividades em campo diferem entre jogadores, é possível que a resistência à fadiga seja variável de acordo com o posicionamento em campo, embora os estudos que realizaram essa investigação apresentem resultados controversos ${ }^{13,22}$. Dessa forma, os objetivos deste estudo foram: a) investigar a existência de diferenças na assimetria de pico de torque, assimetria de trabalho e índice de fadiga isocinética dos extensores e flexores do joelho de jogadores de futebol, de acordo com o posicionamento em campo; e b) determinar se o índice de fadiga dos flexores é superior ao índice de fadiga dos extensores do joelho nesses jogadores.

\section{MÉTODOS}

\section{Amostra}

Foi conduzido um estudo retrospectivo com os dados de avaliações isocinéticas de 164 atletas profissionais de futebol avaliados no período de pré-temporada entre os anos de 2008 e 2011. A amostra foi constituída de 40 atacantes, 27 zagueiros, 23 laterais, 61 meio-campistas e 13 goleiros. A caracterização dos atletas de acordo com o posicionamento em campo encontra-se na tabela 1. Foram excluídos os jogadores submetidos a qualquer cirurgia em membros inferiores no último ano, assim como aqueles que apresentaram alguma lesão musculoesquelética nos membros inferiores nos últimos seis meses. O presente estudo foi aprovado pelo Comitê de Ética em Pesquisa da Universidade (CAAE - 0300.0.203.000-11). 
Tabela 1. Idade, massa corporal e dominância direita em membros inferiores de acordo com o posicionamento em campo do atleta.

\begin{tabular}{c|c|c|c|c|c}
\hline \multirow{2}{*}{ Características } & \multicolumn{5}{|c}{ Posicionamento em campo } \\
\cline { 2 - 6 } & $\begin{array}{c}\text { Atacantes } \\
(\mathbf{n}=\mathbf{4 0})\end{array}$ & $\begin{array}{c}\text { Zagueiros } \\
(\mathbf{n}=\mathbf{2 7})\end{array}$ & $\begin{array}{c}\text { Laterais } \\
\mathbf{( n = 2 3 )}\end{array}$ & $\begin{array}{c}\text { Meio } \\
\text { campistas } \\
(\mathbf{n = 6 1 )}\end{array}$ & $\begin{array}{c}\text { Goleiros } \\
(\mathbf{n}=\mathbf{1 3})\end{array}$ \\
\hline $\begin{array}{c}\text { Idade (anos), } \\
\text { média (DP) }\end{array}$ & $24,8(4,8)$ & $23,4(5,1)$ & $25,2(4,4)$ & $24,2(4,5)$ & $23,6(4,2)$ \\
\hline $\begin{array}{c}\text { Massa corporal } \\
\text { (kg), média (DP) }\end{array}$ & $75,2(7,8)$ & $82,8(7,0)$ & $72,9(6,1)$ & $74,8(6,5)$ & $87,5(6,2)$ \\
\hline $\begin{array}{c}\text { Membro } \\
\text { inferior direito } \\
\text { dominante (\%) }\end{array}$ & 90 & 88,9 & 52,2 & 73,8 & 84,6 \\
\hline
\end{tabular}

$\mathrm{DP}=$ desvio padrão.

\section{Instrumentos e procedimentos}

Todos os atletas tiveram sua massa corporal avaliada por meio de uma balança digital (Filizola S.A. - São Paulo, SP, Brasil) e realizaram uma corrida submáxima de cinco minutos em esteira ergométrica Moviment (Brudden Equipamentos - São Paulo, SP, Brasil) para aquecimento muscular. Um dinamômetro isocinético Biodex 3 Pro (Biodex Medical Systems - Shirley, NY - EUA) foi utilizado para avaliação da força muscular concêntrica dos extensores e flexores do joelho. Os atletas foram posicionados sentados, com o encosto da cadeira do dinamômetro inclinado a uma angulação de $70^{\circ}$ e com o tronco e a pelve estabilizados com cintos do próprio equipamento. O eixo rotacional do dinamômetro foi alinhado ao epicôndilo lateral do fêmur e o acessório específico para avaliação do joelho fixado dois centímetros acima do maléolo medial do tornozelo, conforme indicado pelo fabricante. A amplitude total de movimento utilizada foi de $100^{\circ}$, partindo-se de $110^{\circ}$ de flexão até $10^{\circ}$ de extensão do joelho. $\mathrm{O}$ protocolo de teste consistiu em cinco repetições concêntricas na velocidade angular de $60 \%$ s e 30 repetições na velocidade angular de $300 \%$, sendo o período de repouso entre as séries de 30 segundos. Os extensores e flexores do joelho de ambos os membros inferiores foram avaliados em todos os atletas, iniciando-se sempre pelo membro inferior dominante. Antes do início do teste, os atletas realizaram três repetições em cada velocidade com contrações submáximas, objetivando a familiarização com os procedimentos da avaliação. Em seguida, o torque gerado pelo peso da perna e do pé foi registrado pelo instrumento na posição de $10^{\circ}$ de extensão do joelho. No momento do teste, o atleta recebeu estímulo verbal para realizar força máxima durante os movimentos de extensão e flexão do joelho. Todos os testes foram realizados por dois avaliadores com experiência em avaliações isocinéticas.

\section{Redução dos dados}

A partir do resultado fornecido pelo software (Biodex Advantage Software, v. 1.56) do equipamento, as variáveis de assimetria de pico de torque e trabalho máximo a $60 \%$ s, expressas em porcentagem, foram selecionadas. Além disso, selecionou-se o índice de fadiga dos extensores e flexores do joelho a 300\%s. Esse índice é expresso em porcentagem a partir da diferença na produção de trabalho muscular entre o primeiro e o último terços do total de 30 repetições. Para análise dos índices de assimetria de pico de torque e trabalho foi utilizado o módulo dos valores, com o objetivo de considerar apenas a magnitude da assimetria e não a sua direção.

\section{Análise estatística}

As assimetrias de pico de torque e trabalho máximo, assim como o índice de fadiga dos grupos extensores e flexores do joelho, foram expressos em mediana, percentis 25 e 75 de acordo com o posicionamento em campo dos atletas. Devido a uma assimetria na distribui- ção dos dados, evidenciada por meio de histogramas e pelo teste de Shapiro-Wilk, utilizou-se análise estatística não paramétrica. O teste de Kruskall-Wallis foi utilizado para verificar se havia diferença na assimetria de pico de torque, assimetria de trabalho máximo e no índice de fadiga dos extensores e flexores do joelho entre os grupos de jogadores (atacantes, zagueiros, laterais, meio-campistas e goleiros). O teste de Wilcoxon foi realizado a fim de verificar a existência de diferença entre o índice de fadiga de extensores e flexores para os membros inferiores dominante e não dominante. Um nível de significância (a) de 0,05 foi utilizado para análise dos dados.

\section{RESULTADOS}

A mediana e os percentis 25 e 75 das variáveis assimetria de pico de torque e trabalho máximo dos extensores e flexores do joelho de acordo com a posição em campo dos jogadores encontram-se na tabela 2. Além disso, a tabela 3 apresenta os valores descritivos do índice de fadiga de extensores e flexores do joelho de acordo com a posição em campo.

Tabela 2. Análise descritiva das variáveis de assimetria de pico de torque e trabalho máximo de acordo com o posicionamento em campo.

\begin{tabular}{|c|c|c|c|c|}
\hline \multirow{2}{*}{$\begin{array}{l}\text { Posicionamento } \\
\text { em campo }\end{array}$} & \multicolumn{2}{|c|}{$\begin{array}{c}\text { Assimetria de } \\
\text { pico de torque (\%) }\end{array}$} & \multicolumn{2}{|c|}{$\begin{array}{c}\text { Assimetria de } \\
\text { trabalho máximo (\%) }\end{array}$} \\
\hline & Extensor & Flexor & Extensor & Flexor \\
\hline \multicolumn{5}{|l|}{ Atacantes $(n=40)$} \\
\hline Mediana & 7,50 & 6,50 & 8,15 & 5,40 \\
\hline Percentil 25 & 4,93 & 3,40 & 3,78 & 2,20 \\
\hline Percentil 75 & 12,55 & 11,23 & 12,08 & 10,00 \\
\hline \multicolumn{5}{|l|}{ Zagueiros $(n=27)$} \\
\hline Mediana & 10,10 & 8,90 & 7,50 & 8,70 \\
\hline Percentil 25 & 3,90 & 3,20 & 2,70 & 3,50 \\
\hline Percentil 75 & 17,60 & 14,30 & 15,90 & 15,00 \\
\hline \multicolumn{5}{|l|}{ Laterais $(n=23)$} \\
\hline Mediana & 8,20 & 7,50 & 7,10 & 6,20 \\
\hline Percentil 25 & 3,90 & 4,00 & 4,10 & 4,80 \\
\hline Percentil 75 & 12,30 & 14,30 & 13,50 & 12,50 \\
\hline
\end{tabular}

\section{Meio-campistas $(n=61)$}

\begin{tabular}{c|c|c|cc}
\hline Mediana & 8,00 & 8,40 & 7,40 & 7,90 \\
\hline Percentil 25 & 4,30 & 4,55 & 3,85 & 3,10 \\
\hline Percentil 75 & 13,45 & 13,95 & 13,50 & 13,80 \\
\hline Goleiros (n = 13) & & & & 4,70 \\
\hline Mediana & 5,30 & 6,00 & 9,10 & 1,55 \\
\hline Percentil 25 & 3,05 & 2,50 & 4,00 & 10,50 \\
\hline Percentil 75 & 13,45 & 9,35 & 11,50 & 7,40 \\
\hline Total (n = 164) & & & & 3,03 \\
\hline Mediana & 7,75 & 8,10 & 7,65 & 12,68 \\
\hline Percentil 25 & 4,33 & 3,60 & 3,73 & 12,88 \\
\hline Percentil 75 & 13,18 & 12,75 & &
\end{tabular}

O teste de Kruskall-Wallis não evidenciou diferença significativa entre os grupos de jogadores dos cinco posicionamentos em campo para a assimetria de pico de torque dos extensores $(H(4)=1,40, p=0,85)$ e flexores $(H(4)=5,33, p=0,26)$; assimetria de trabalho máximo dos extensores $(H(4)=0,15, p=1,00)$ e flexores $(H(4)=5,83, p=0,21)$; índice de fadiga dos extensores do membro inferior dominante $(H(4)=4,98$, $p=0,29)$ e não dominante $(H(4)=0,05, p=1,00)$; índice de fadiga dos 
Tabela 3. Análise descritiva do índice de fadiga de acordo com o posicionamento em campo.

\begin{tabular}{|c|c|c|c|c|}
\hline & \multicolumn{4}{|c|}{ Índice de fadiga (\%) } \\
\hline & \multicolumn{2}{|c|}{ Dominante } & \multicolumn{2}{|c|}{ Não dominante } \\
\hline & Extensor & Flexor & Extensor & Flexor \\
\hline \multicolumn{5}{|c|}{ Atacantes $(n=40)$} \\
\hline Mediana & 51,25 & 59,05 & 51,20 & 57,60 \\
\hline Percentil 25 & 47,15 & 51,30 & 45,43 & 50,88 \\
\hline Percentil 75 & 53,88 & 63,38 & 57,70 & 63,03 \\
\hline \multicolumn{5}{|c|}{ Zagueiros $(n=27)$} \\
\hline Mediana & 50,40 & 55,50 & 50,20 & 52,00 \\
\hline Percentil 25 & 44,90 & 45,50 & 46,70 & 47,70 \\
\hline Percentil 75 & 54,90 & 60,00 & 56,30 & 60,30 \\
\hline \multicolumn{5}{|c|}{ Laterais $(n=23)$} \\
\hline Mediana & 49,40 & 53,40 & 50,80 & 62,30 \\
\hline Percentil 25 & 45,90 & 48,90 & 45,00 & 50,30 \\
\hline Percentil 75 & 52,00 & 65,60 & 56,40 & 65,80 \\
\hline \multicolumn{5}{|c|}{ Meio-campistas $(n=61)$} \\
\hline Mediana & 49,50 & 58,20 & 49,50 & 58,00 \\
\hline Percentil 25 & 45,75 & 50,25 & 46,55 & 48,20 \\
\hline Percentil 75 & 53,00 & 65,45 & 55,70 & 63,15 \\
\hline \multicolumn{5}{|c|}{ Goleiros $(n=13)$} \\
\hline Mediana & 51,20 & 64,10 & 51,50 & 62,20 \\
\hline Percentil 25 & 49,90 & 48,60 & 44,55 & 52,20 \\
\hline Percentil 75 & 55,15 & 70,80 & 55,65 & 67,70 \\
\hline \multicolumn{5}{|l|}{ Total $(n=164)$} \\
\hline Mediana & 50,45 & 57,85 & 50,35 & 57,60 \\
\hline Percentil 25 & 46,45 & 50,53 & 46,33 & 50,13 \\
\hline Percentil 75 & 53,50 & 64,73 & 55,03 & 63,38 \\
\hline
\end{tabular}

flexores do membro inferior dominante $(H(4)=3,58, p=0,47)$ e não dominante $(H(4)=5,33, p=0,26)$.

O teste de Wilcoxon evidenciou diferença entre o índice de fadiga de extensores e flexores do joelho no membro inferior dominante $(z=-8,76, p<0,01)$ e não dominante $(z=-8,04, p<0,01)$. De acordo com os dados descritivos apresentados na tabela 3 , observa-se que a mediana do índice de fadiga dos flexores é superior ao dos extensores no membro inferior dominante e não dominante.

\section{DISCUSSÃO}

Este estudo não identificou diferença na assimetria de pico de torque, assimetria de trabalho máximo e índice de fadiga isocinética dos extensores e flexores do joelho entre atletas profissionais de futebol de acordo com o posicionamento em campo (atacantes, zagueiros, laterais, meio-campistas e goleiros). Em relação à diferença do índice de fadiga entre grupos musculares, observou-se que os flexores apresentaram maior fadiga do que os extensores do joelho.

A ausência de diferença na assimetria de pico de torque e trabalho máximo entre os cinco posicionamentos em campo está de acordo com os resultados de outros dois estudos ${ }^{1,22}$. Esse resultado indica que não foi evidenciada a existência de uma maior assimetria de força nos laterais, quando comparados aos jogadores das demais posições. Tourny-Chollet et al. ${ }^{14}$ evidenciaram maior assimetria de pico de torque nos meio-campistas e atacantes em comparação aos defensores. No entanto, a amostra total desse estudo foi constituída por apenas 21 jogadores, os quais foram agrupados somente em três categorias de posicionamento em campo (atacantes, meio-campistas e defensores). No presente estudo, a grande amostra utilizada indica que a posição do jogador não influencia diferentemente a capacidade de geração de força dos flexores e extensores de joelho entre membros inferiores.

Embora cada posicionamento em campo exija movimentos específicos, é possível que o treinamento de força realizado diariamente nos clubes esportivos envolvidos no presente estudo seja semelhante para todos os jogadores, o que pode justificar a ausência de diferença entre os jogadores dos diversos posicionamentos. Esse fato pode explicar os percentuais relativamente baixos de assimetria encontrados na maioria dos jogadores da amostra (mediana entre 5,4 e 10,1\%), independente da posição em campo. Evidências indicam que uma assimetria de força é preditiva de lesões musculoesqueléticas apenas quando a diferença entre os membros inferiores é superior a 10\%3,10. Dessa forma, apesar de uma partida de futebol envolver a execução de movimentos assimétricos e variados de acordo com o posicionamento em campo, o treinamento de força realizado rotineiramente pelos atletas deste estudo parece ser suficiente para prevenir a ocorrência de possíveis assimetrias entre os membros inferiores dos jogadores de todos os posicionamentos.

O índice de fadiga dos extensores e flexores do joelho também não foi diferente entre os cinco posicionamentos em campo. Essa variável é pouco investigada na literatura, sendo que foram encontrados apenas dois estudos com objetivos similares ao nosso ${ }^{13,22}$. Embora no estudo de Weber et al. ${ }^{13}$ os jogadores tenham sido divididos em categorias diferentes daquelas utilizadas no presente estudo, o índice de fadiga também não foi diferente de acordo com o posicionamento em campo. No entanto, Goulart et al.22 evidenciaram que os goleiros possuíam maior percentual de fadiga dos extensores do joelho quando comparados aos jogadores dos demais posicionamentos (zagueiros, laterais, volantes, meio-campistas e atacantes). Nesse mesmo estudo, os atletas que atuavam em posicionamentos laterais apresentaram maior índice de fadiga dos flexores do joelho quando comparados aos atacantes ${ }^{22}$. Além disso, a mediana referente aos índices de fadiga dos extensores e flexores variou de 64,8 a $77,3 \%$, enquanto no presente estudo esses valores foram de 49,4 a $64,1 \%$. A diferença nesses valores sugere que os atletas investigados no presente estudo podem ter sido mais expostos a treinamentos direcionados ao ganho de resistência das musculaturas investigadas. Esse treinamento de resistência é, possivelmente, similar entre todos os jogadores, visto que não houve diferença no percentual de fadiga de acordo com os cinco posicionamentos em campo.

Assim como no presente estudo, outros autores determinaram o índice de fadiga a partir da relação entre o trabalho realizado no primeiro e último terços de um total de 30 repetições dos movimentos de extensão e flexão do joelho na velocidade de $300^{\circ} / \mathrm{s}^{13,22}$. No entanto, existem outras maneiras de investigar a fadiga isocinética, sendo que não há um consenso na literatura sobre o método mais adequado para calcular a fadiga e o melhor protocolo de teste a ser utilizado 17,23. Além disso, existem outros métodos para avaliação da fadiga muscular, como a eletromiografia ${ }^{24}$. Dessa forma, embora o índice de fadiga não tenha sido diferente entre os atletas dos diversos posicionamentos em campo, outros métodos de avaliação da fadiga muscular poderiam produzir resultados distintos daqueles observados no presente estudo.

Este estudo evidenciou que a musculatura flexora do joelho de atletas profissionais de futebol possui um índice de fadiga significativamente maior do que aquele encontrado para a musculatura extensora. O maior índice de fadiga dos flexores do joelho observado no presente estudo pode ser explicado pela maior proporção de fibras do tipo II nos isquiossurais em comparação ao 
quadríceps, visto que as fibras desse tipo são menos resistentes à fadiga do que as fibras do tipo ${ }^{25}$. Além disso, o maior índice de fadiga dos flexores do joelho pode favorecer a ocorrência de lesões nessa musculatura. Pesquisas têm evidenciado uma maior incidência de lesões por estiramento em isquiossurais quando comparada àquela observada na musculatura extensora ${ }^{20,21}$. Uma vez que a musculatura que apresentou um maior índice da fadiga foi aquela que possui maior incidência de lesões musculares, futuros estudos devem investigar a relação entre o índice de fadiga isocinético dos isquiossurais e a incidência de lesões dessa musculatura.

Os valores de mediana, percentis 25 e 75 encontrados para as variáveis isocinéticas deste estudo poderão ser utilizados como referência para avaliações pré-temporada de atletas profissionais de futebol. Jogadores avaliados com o protocolo de teste deste estudo que apresentarem assimetria na variável pico de torque ou trabalho máximo para os flexores ou extensores do joelho acima da mediana reportada podem beneficiar-se de um programa direcionado para correção dessas assimetrias. Da mesma forma, jogadores que apresentarem índice de fadiga dos extensores ou flexores acima da mediana relatada podem ser encaminhados para um treinamento focado na melhora da resistência muscular. Além disso, os percentis 25 e 75 podem ser utilizados para identificar os atletas nos extremos da distribuição de assimetria e índice de fadiga. Esses percentis podem auxiliar na identificação de atletas com maior susceptibilidade a lesões, bem como guiar programas de treinamento. Ressalta-se que as variáveis isocinéticas são consideradas somente um dos fatores para o direcionamento de programas de prevenção, uma vez que lesões musculoesqueléticas possuem natureza multifatorial. Dessa forma, os valores das variáveis isocinéticas encontrados, juntamente com outras variáveis de uma avaliação pré-temporada, contribuirão para o desenvolvimento de programas preventivos.

A interpretação dos achados deste estudo deve levar em consideração que a avaliação dos grupos musculares por meio do dinamômetro isocinético é realizada em posições padronizadas, com velocidades angulares constantes e movimentos que podem não refletir completamente o desempenho muscular durante o gesto esportivo ${ }^{26}$. Contudo, ressalta-se que as variáveis investigadas são comumente reportadas como um dos fatores associados a lesões esportivas em jogadores de futebol. Além disso, estudos indicam que variáveis isocinéticas resultantes de uma avaliação muscular excêntrica também possuem associação com história prévia ${ }^{27}$ e predição de lesão muscular². No entanto, no presente estudo, as avaliações isocinéticas foram realizadas apenas no modo concêntrico. Dessa forma, estudos futuros devem investigar variáveis de testes excêntricos considerando uma divisão dos jogadores de acordo com a posição em campo, como realizado neste estudo.

\section{CONCLUSÃO}

A partir dos resultados encontrados, conclui-se que assimetria de pico de torque, assimetria de trabalho máximo e índice de fadiga dos extensores e flexores do joelho, comumente associados à ocorrência de lesões, não apresentam diferenças de acordo com a posição em campo do atleta. Além disso, observou-se que o índice de fadiga dos flexores é superior ao dos extensores do joelho em atletas profissionais de futebol.

Todos os autores declararam não haver qualquer potencial conflito de interesses referente a este artigo.

\section{REFERÊNCIAS}

1. Magalhaes J, Oliveira J, Ascensao A, Soares J. Concentric quadriceps and hamstrings isokinetic strength in volleyball and soccer players. J Sports Med Phys Fitness 2004;44:119-25.

2. Fousekis $K$, Tsepis $E$, Vagenas $G$. Multivariate isokinetic strength asymmetries of the knee and ankle in professional soccer players. J Sports Med Phys Fitness 2010;50:465-74.

3. Grace TG, Sweetser ER, Nelson MA, Ydens LR, Skipper BJ. Isokinetic muscle imbalance and knee-joint injuries. A prospective blind study. J Bone Joint Surg Am 1984;66:734-40.

4. Wyatt MP, Edwards AM. Comparison of Quadriceps and Hamstring Torque Values during Isokinetic Exercise. J Orthop Sports Phys Ther 1981;3:48-56.

5. Knapik JJ, Bauman CL, Jones BH, Harris JM, Vaughan L. Preseason strength and flexibility imbalances associated with athletic injuries in female collegiate athletes. Am J Sports Med 1991;19:76-81.

6. Croisier JL, Ganteaume S, Binet J, Genty M, Ferret JM. Strength imbalances and prevention of hamstring injury in professional soccer players: a prospective study. Am J Sports Med 2008;36:1469-75.

7. Devan MR, Pescatello LS, Faghri P, Anderson J. A Prospective Study of Overuse Knee Injuries Among Female Athletes With Muscle Imbalances and Structural Abnormalities. J Athl Train 2004;39:263-7.

8. Ekstrand J, Gillquist J. Soccer injuries and their mechanisms: a prospective study. Med Sci Sports Exerc 1983;15:267-70.

9. Rahnama N, Lees A, Bambaecichi E. Comparison of muscle strength and flexibility between the preferred and non-preferred leg in English soccer players. Ergonomics 2005;48:1568-75.

10. Mognoni P, Narici MV, Sirtori MD, Lorenzelli F. Isokinetic torques and kicking maximal ball velocity in young soccer players. J Sports Med Phys Fitness 1994;34:357-61.

11. Capranica L, Cama G, Fanton F, Tessitore A, Figura F. Force and power of preferred and non-preferred leg in young soccer players. J Sports Med Phys Fitness 1992;32:358-63.

12. Bennell K, Wajswelner H, Lew P, Schall-Riaucour A, Leslie S, Plant D, et al. Isokinetic strength testing does not predict hamstring injury in Australian Rules footballers. Br J Sports Med 1998;32:309-14.

13. Weber FS, Silva BGC, Radaelli R, Paiva C, Pinto RS. Avaliação isocinética em jogadores de futebol profissional e comparação do desempenho entre as diferentes posições ocupadas em campo. Rev Bras Med Esporte 2010;16:264-8

14. Tourny-Chollet C, Leroy D, Léger H, Beuret-Blanquart F. Isokinetic knee muscle strength of soccer players according to their position. Isokinet Exerc Sci 2000;8:187-93.
15. Dvir Z. Isokinetics - Muscle Testing, Interpretation and Clinical Applications. 2nd ed. London: Chuchill Livingstone; 2004.

16. Nawoczenski DA, Neumann DA. Princípios de Biomecânica. In: Neumann DA, editor. Cinesiologia do aparelho musculoesquelético: fundamentos para reabilitação física. Rio de Janeiro: Guanabara Koogan; 2006.

17. Saenz A, Avellanet M, Hijos E, Chaler J, Garreta R, Pujol E, et al. Knee isokinetic test-retest: a multicentre knee isokinetic test-retest study of a fatigue protocol. Eur J Phys Rehabil Med 2010;46:81-8.

18. Alentorn-Geli E, Myer GD, Silvers HJ, Samitier G, Romero D, Lazaro-Haro C, et al. Prevention of non-contact anterior cruciate ligament injuries in soccer players. Part 1: Mechanisms of injury and underlying risk factors. Knee Surg Sports Traumatol Arthrosc 2009;17:705-29.

19. Woods C, Hawkins RD, Maltby S, Hulse M, Thomas A, Hodson A. The Football Association Medical Research Programme: an audit of injuries in professional football - analysis of hamstring injuries. $B$ J Sports Med 2004;38:36-41.

20. Ekstrand J, Hagglund M, Walden M. Epidemiology of muscle injuries in professional football (soccer) Am J Sports Med 2011;39:1226-32

21. Fousekis K, Tsepis E, Poulmedis P, Athanasopoulos S, Vagenas G. Intrinsic risk factors of non-contact quadriceps and hamstring strains in soccer: a prospective study of 100 professional players. $\mathrm{Br}$ Sports Med 2011;45:709-14.

22. Goulart LF, Dias RMR, Altimari LR. Força isocinética de jogadores de futebol categoria sub-20:comparação entre diferentes posições de jogo. Rev Bras Cineantropom Desempenho Hum 2007;9:165-9.

23. Manou V, Arseniou P, Gerodimos P, Kellis S. Test-retest reliability of an isokinetic muscle endurance test. Isokinet Exerc Sci 2002;10:177-81.

24. Rampinini E, Bosio A, Ferraresi I, Petruolo A, Morelli A, Sassi A. Match-related fatigue in soccer players. Med Sci Sports Exerc 2011;43:2161-70.

25. Garrett WE, Jr., Califf JC, Bassett FH, III. Histochemical correlates of hamstring injuries. Am J Sports Med 1984;12:98-103.

26. Chan K. Isokinetic in Rehabilitation. In: Puddu G, Giombini A, Selvanetti A, editors. Rehabilitation of sports injury: current concepts. Berlin: Springer; 2001.

27. Dauty M, Potiron-Josse M, Rochongar P. Identification of previous hamstring muscle injury by isokinetic concentric and eccentric torque measurement in elite soccer player. Isokinet Exerc Sci 2003;1 1:139-44. 